\title{
The Impact of Gross Motor Function on the Oral Health-Related Quality of Life in Young Adults with Cerebral Palsy in Saudi Arabia
}

\author{
Sharat Chandra Pani $\mathbb{D}^{1},{ }^{1}$ Saja Fahad AlEidan, ${ }^{2}$ Rawan Nasser AlMutairi, ${ }^{2}$ \\ AlJawharah Ali AlAbsi, ${ }^{2}$ Dalal Nasser AlMuhaidib, ${ }^{2}$ Hessa Faisal AlSulaiman, \\ and Najd Waleed AlFraih ${ }^{2}$ \\ ${ }^{1}$ Schulich School of Dentistry and Pharmacy, Western University, London, ON, Canada \\ ${ }^{2}$ College of Dentistry, Riyadh Elm University, Riyadh, Saudi Arabia \\ Correspondence should be addressed to Sharat Chandra Pani; spani@uwo.ca
}

Received 8 November 2019; Revised 29 January 2020; Accepted 4 February 2020; Published 28 February 2020

Academic Editor: Alessandro Leite Cavalcanti

Copyright (C) 2020 Sharat Chandra Pani et al. This is an open access article distributed under the Creative Commons Attribution License, which permits unrestricted use, distribution, and reproduction in any medium, provided the original work is properly cited.

\begin{abstract}
Background. There is evidence that gross motor function impacts the health-related quality of life of young adults with cerebral palsy. This study aimed to assess gross motor function, oral health and oral health-related quality of life (OHRQoL), and the relationship between them in young adults with cerebral palsy. Methods. The sample comprised 46 individuals aged between 13 and 17 years with Gross Motor Function Classification Scores (GMFCS) ranging from level I to level III. The individuals and their parents were administered an Arabic version of the child perception questionnaire for adolescents. Parental and child perception scores, DMFT, and gingival index were compared across GMFCS levels using the one-way ANOVA and Scheffe's post hoc test. Results. Children with level III GMFCS had a significantly higher child perception score (CPQ) and parental perception score (PPQ) than those with level I or level II scores. There was a significant association between function (GMFCS) and the CPQ score in children $(p=0.016)$. No significant associations were found between the CPQ score and either dental caries (DMFT) or gingival bleeding (GI) scores. Children with GMFCS level III had a significantly higher DMFT $(p=0.002)$ and GI $(p=0.001)$ scores. Conclusion. Motor function has a significant impact on both the oral health and the OHRQoL of adolescents and young adults with spastic cerebral palsy.
\end{abstract}

\section{Introduction}

Cerebral palsy $(\mathrm{CP})$ is not a specific disease entity but rather a collection of disabling disorders caused by insult and permanent damage to the brain in the prenatal and perinatal periods, during which time the central nervous system is still maturing [1]. This disability might involve muscle weakness, stiffness or paralysis, poor balance or irregular gait, and uncoordinated or involuntary movements [1]. It has been reported that cerebral palsy was one of the most common neurological disorders among Saudi children with a prevalence of 42 per 10000 live births $(0.42 \%)$ [2].

The health-related quality of life (HRQoL) of adolescents and young adults with cerebral palsy has recently received attention in literature $[3,4]$. Researchers have pointed out that the level of functional activity of the individual with $\mathrm{CP}$ plays an important role in the overall perception of their HRQoL [5-7]. With growing emphasis on the inclusion of individuals with CP into mainstream activities, it has been pointed out that it is important to be able to measure both function $[8,9]$ and HRQoL of these individuals [3].

Oral health-related quality of life (OHRQoL) is a multidimensional construct that includes a subjective evaluation of the individual's oral health, functional well-being, emotional well-being, expectations and satisfaction with care, and sense of self. It has wide-reaching applications in survey and clinical research [10-13]. The OHRQoL is an integral part of general health and well-being. There have been several scales specifically designed to quantitatively assess the OHRQoL in children and young adults $[10,13-16]$. The 
CPQ11-14 has proven to be a good predictor of quality of life among adolescents and young adults [7].

The OHRQoL of individuals with CP has only recently been receiving attention in literature with studies looking into the OHRQoL of both children with CP and their caregivers $[17,18]$. The traditional method of studying the OHRQoL in children with CP has focused on the experiences of the parents $[17,19]$. There is, however, some evidence to suggest that significant variations exist in the perception of OHRQoL reported by parents when compared to the values reported by their children with CP [20].

Function plays an important role in the overall healthrelated quality of life of individuals with cerebral palsy [20-22]. There are several methods of evaluating motor function in children with cerebral palsy of which the gross motor function classification system (GMFCS) is widely accepted. The revised version of the GMFCS has been found to be an acceptable tool for assessment of motor function in adolescents [7]. While there is growing evidence on the role of function on HRQoL of individuals with CP, there is little known about the role it plays on the OHRQoL. Given the relationship between function and physical development, the age study of function in young adults necessitates the examination of a relatively narrow age range of individuals.

This study aimed to assess gross motor function, oral health and oral health-related quality of life (OHRQoL), and the relationship between them in young adults with $\mathrm{CP}$ aged between 13 and 17 years attending a rehabilitation center in Riyadh, Saudi Arabia.

\section{Materials and Methods}

2.1. Ethical Approval. The study was registered with the research center of the Riyadh Elm University (FUGRP/2018/ 140 ), and ethical approval was obtained from the Institutional Review Board of the Institution (RC/IRB/2018/1054). Ethical approval was also obtained from the ethical committee of the Prince Sultan Humanitarian City. Written informed consent was obtained from all parents and participants in the study, and assent was obtained from each participant before the administration of the questionnaire and oral examination.

2.2. Study Design. A cross-sectional study design was used to measure the OHRQoL in individuals with CP aged between 13 and 17 years and their parents.

2.3. Recording of Variables. A validated Arabic Version of the Parental Perception Questionnaire and Child Perception Questionnaire was used for the determination of OHRQoL [15]. The questionnaire comprised a parental component that was filled in by the parent and a child component that was filled in by the examiner after speaking to the individual with cerebral palsy. Each component comprised 16 questions divided into four domains: oral symptoms, functional limitations, emotional, and social well-being. In addition, the questionnaire comprises an 8-question family impact score that is answered by the parent [23-25]. The total number of decayed, missing, and filled permanent teeth (DMFT) and the gingival index were recorded to assess caries levels and gingival status, respectively. The teeth were examined using WHO category II criteria: clinical examination with dental unit light and without radiographs. Examiners were calibrated for the use of the recording form and for the detection of dental caries. A total of three examiners examined the patients (NaF, SaE, and $\mathrm{AaA})$. The overall kappa for the total number of decayed, missing, and filled was 0.871 among three examiners. No kappa measurements were taken for gingival bleeding scores. However, as recommended by the WHO, examiners were trained for the measurement of gingival problems.

\subsection{Sample Selection and Administration of Questionnaire.} The sample comprised patients who were attending the outpatient clinics of the Prince Sultan Humanitarian City, Riyadh. The patients were aged between 13 and 17 years and were diagnosed with mild to moderate cerebral palsy and had been classified by the pediatric neurologist at the center as being either level I, level II, or level III on the Gross Motor Function Classification System (GMFCS). All participants had to be able to walk independently (at least with a waking aid). Only children with spastic monoplegia, hemiplegia or spastic diplegia were included in the study. Children with visual or hearing impairments, those with other forms of $\mathrm{CP}$, and those with any additional disease were excluded from the sample.

2.5. Statistical Analyses. The one-way ANOVA and Scheffe's post hoc test were used to compare OHRQoL (CPQ or PPQ), dental caries (DMFT), and gingival status (GI) scores among children with different levels of function (GMFCS score). All statistical analyses were performed using IBM-SPSS v25 data processing software (IBM Corp. Armonk NY, USA), and the alpha for all tests was set at $p<0.05$.

\section{Results}

The sample comprised 45 adolescents (21 male 25 female) with cerebral palsy reporting for outpatient dental care at the Prince Sultan Humanitarian City. The adolescents ranged between 13 and 17 years of age (mean age 15.2, SD \pm 2.9 years). Diplegia was the most common form of limb involvement seen followed by monoplegia and hemiplegia (Table 1). When the CP and level of movement of the participants were compared, it was observed that there were fewer children with hemiplegia $(n=9)$ than with diplegia $(n=19)$ or monoplegia $(n=18)$. It was also observed that a majority of the sample population $(n=31)$ were on some forms of long-term medication. Almost all the children in the sample $(n=42)$ had a history of surgery (Table 1$)$.

Most of the primary guardians were mothers $(n=35)$, while fathers $(n=7)$ and caregivers $(n=4)$ were less frequent as the primary caregiver. The demographic profile of the families interviewed was a mixture of the different sociodemographic groups (Table 2). When oral health-related quality of life and parental perception of OHRQoL were 
TABLE 1: Description of the study population.

\begin{tabular}{|c|c|c|c|c|c|c|c|}
\hline & & \multicolumn{2}{|c|}{ Male } & \multicolumn{2}{|c|}{ Female } & \multirow{2}{*}{ Total } & \multirow{2}{*}{$\%$} \\
\hline & & $N$ & $\%$ & $N$ & $\%$ & & \\
\hline Limb & Monoplegia & 10 & 47.6 & 8 & 32.0 & 18 & 39.1 \\
\hline \multirow{2}{*}{ Involvement } & Hemiplegia & 6 & 28.6 & 3 & 12.0 & 9 & 19.6 \\
\hline & Diplegia & 5 & 23.8 & 14 & 56.0 & 19 & 41.3 \\
\hline \multirow{2}{*}{ Medication } & No long-term medication & 5 & 23.8 & 10 & 40.0 & 15 & 32.6 \\
\hline & Long-term medication & 16 & 76.2 & 15 & 60.0 & 31 & 67.4 \\
\hline \multirow{2}{*}{ History of surgery } & No & 2 & 9.5 & 2 & 8.0 & 4 & 8.7 \\
\hline & Yes & 19 & 91.5 & 23 & 92.0 & 42 & 91.3 \\
\hline \multirow{3}{*}{ GMFCS score } & Level I & 9 & 42.8 & 8 & 32.0 & 17 & 36.9 \\
\hline & Level II & 7 & 33.4 & 7 & 28.0 & 14 & 30.5 \\
\hline & Level III & 5 & 23.8 & 10 & 40.0 & 15 & 32.6 \\
\hline
\end{tabular}

TABLE 2: Sociodemographic profile of the population.

\begin{tabular}{|c|c|c|c|}
\hline & & Count & Column $N(\%)$ \\
\hline \multirow{5}{*}{ Monthly family income } & $<5000 \mathrm{SR}$ & 6 & 13.0 \\
\hline & 5000SR-10000SR & 13 & 28.3 \\
\hline & 10000SR-20000SR & 18 & 39.1 \\
\hline & 20000-40000SR & 7 & 15.2 \\
\hline & $<40000 \mathrm{SR}$ & 2 & 4.3 \\
\hline \multirow{4}{*}{ Education of father } & Elementary school & 13 & 28.3 \\
\hline & High school & 18 & 39.1 \\
\hline & Graduate degree & 13 & 28.3 \\
\hline & Postgraduate degree & 2 & 4.3 \\
\hline \multirow{4}{*}{ Education of mother } & Elementary school & 13 & 28.3 \\
\hline & High school & 19 & 41.3 \\
\hline & Graduate degree & 14 & 30.4 \\
\hline & Postgraduate degree & 0 & 0.0 \\
\hline \multirow{2}{*}{ Employment status of father } & Unemployed & 19 & 41.3 \\
\hline & Employed & 27 & 58.7 \\
\hline \multirow{2}{*}{ Employment status of mother } & Unemployed & 35 & 76.1 \\
\hline & Employed & 11 & 23.9 \\
\hline
\end{tabular}

examined, it was observed that there was a significant increase in child perception scores with a decrease in the level of function. The same was true for parental perception scores (Table 3). Scheffe's post hoc test showed that there was a significant difference between children with level I and level III GMFCS scores $(p<0.05)$. Scheffe's post hoc test showed that there were no significant differences in the child perception scores between children with level I and level II GMFCS score $(p=0.171)$ or between children with level II and level III GMFCS scores $(p=0.092)$. When parental perceptions scores were compared, it was observed that there was no significant difference between parents of children with level I or level II GMFCS scores $(p=0.566)$. However, parents of children with GMFCS score III showed a significantly higher score on the parental perception questionnaire $(p<0.05)$.

When the oral health of children was compared among children with different levels of function, it was observed that the one-way ANOVA showed that there were significant differences in both DMFT and GI scores. The post hoc tests revealed that there were no significant differences in DMFT between individuals with level I and level II function $(p=0.670)$, but individuals with level III functional impairment had a significantly higher DMFT score $(p<0.05)$. When the GI scores were compared, it was observed that individuals with level II had significantly higher sores than those with level I function $(p<0.05)$ but significantly lower GI scores than those with level III function $(p<0.05)$ (Table 4).

\section{Discussion}

The oral health of children and young adults with cerebral palsy has been studied since 1970s. However, it is only recently that studies have sought to look at the impact of that impaired oral health on the quality of life of these individuals $[15,17,19]$. This study sought to assess the impact of overall motor function and oral health on the oral health-related quality of life of individuals with cerebral palsy in Saudi Arabia.

Saudi Arabia has a documented history of high dental caries in children and adolescents, even in the absence of underlying systemic disease [23]. There is also documented evidence that children and adolescents in Saudi Arabia have poor oral hygiene practices [24]. It was for this reason that the study chose not to include a control group of CP-free 
TABLE 3: Child and parental perception of OHRQoL according to the level of function.

\begin{tabular}{|c|c|c|c|c|c|c|c|}
\hline & & \multirow{2}{*}{ Mean } & \multirow{2}{*}{ Std. deviation } & \multicolumn{2}{|c|}{ 95\% confidence interval for mean } & \multirow{2}{*}{$F^{*}$} & \multirow{2}{*}{ Sig } \\
\hline & & & & Lower bound & Upper bound & & \\
\hline \multirow{3}{*}{ CPQ } & GMFCS level I ${ }^{\mathrm{a}}$ & 35.0588 & 9.74981 & 30.0459 & 40.0717 & \multirow{3}{*}{9.065} & \multirow{3}{*}{$0.001^{* *}$} \\
\hline & GMFCS level $\mathrm{II}^{\mathrm{ab}}$ & 40.9286 & 8.63325 & 35.9439 & 45.9133 & & \\
\hline & GMFCS level III $^{\mathrm{b}}$ & 47.8000 & 6.42762 & 44.2405 & 51.3595 & & \\
\hline \multirow{3}{*}{ PPQ } & GMFCS level I ${ }^{\mathrm{a}}$ & 36.7647 & 9.37103 & 31.9466 & 41.5828 & \multirow{3}{*}{6.859} & \multirow{3}{*}{$0.003^{* *}$} \\
\hline & GMFCS level $\mathrm{II}^{\mathrm{a}}$ & 40.2143 & 8.14464 & 35.5117 & 44.9169 & & \\
\hline & GMFCS level III $^{\mathrm{b}}$ & 48.2000 & 8.90586 & 43.2681 & 53.1319 & & \\
\hline
\end{tabular}

${ }^{*}$ Calculated using one-way ANOVA. ${ }^{* *}$ Differences significant at $p<0.05$. ${ }^{\text {a,b }}$ Differences in superscript indicate significant difference at $p<0.05$ when compared using Scheffe's post hoc test.

TABLE 4: Impact of functional ability on oral health.

\begin{tabular}{|c|c|c|c|c|c|c|c|}
\hline \multirow{2}{*}{ GMFCS } & & \multirow{2}{*}{ Mean } & \multirow{2}{*}{ Std. deviation } & \multicolumn{2}{|c|}{$95 \%$ confidence interval for mean } & \multirow{2}{*}{$F^{*}$} & \multirow{2}{*}{ Sig } \\
\hline & & & & Lower bound & Upper bound & & \\
\hline \multirow{3}{*}{ DMFT } & Level I ${ }^{\mathrm{a}}$ & 1.1176 & 1.40900 & 0.3932 & 1.8421 & \multirow{3}{*}{7.342} & \multirow{3}{*}{$0.002^{* *}$} \\
\hline & Level II ${ }^{\mathrm{a}}$ & 1.7143 & 2.05421 & 0.5282 & 2.9004 & & \\
\hline & Level III ${ }^{\mathrm{b}}$ & 3.5333 & 2.03072 & 2.4088 & 4.6579 & & \\
\hline \multirow{3}{*}{ GI } & Level I $^{\mathrm{a}}$ & 4.7647 & 3.63197 & 2.8973 & 6.6321 & \multirow{3}{*}{8.218} & \multirow{3}{*}{$0.001^{* *}$} \\
\hline & Level $\mathrm{II}^{\mathrm{b}}$ & 7.2143 & 4.11710 & 4.8371 & 9.5914 & & \\
\hline & Level III ${ }^{\mathrm{c}}$ & 10.4000 & 4.06729 & 8.1476 & 12.6524 & & \\
\hline
\end{tabular}

${ }^{*}$ Calculated using one-way ANOVA. ${ }^{* *}$ Differences significant at $p<0.05 .{ }^{\mathrm{a}, \mathrm{b}, \mathrm{c}}$ Differences in superscript indicate significant difference at $p<0.05$ when compared using Scheffe's post hoc test.

children and adolescents' age and gender matched to the population.

The role of motor function in the overall health-related quality of life has received considerable attention in recent literature $[3,25,26]$. The GMFCS is a validated tool that has been used repeatedly as a measure of motor function in individuals with cerebral palsy [19]. The decision to limit the inclusion criteria in the present study up to level III GMFCS was based on the fact that previous studies have shown individuals with GMFCS level IV and level V to have a compromised motor function that would require assistance $[6,19,25]$.

Studies have also shown a negative correlation between the GMFCS score and the overall activity participation $[3,5,27]$. The fact that the results of our study found a similar relationship with CPQ scores, where there was a worse OHRQoL with increasing GMFCS level, seems to highlight the role function in oral health. This finding is further substantiated by the trend of higher dental caries and gingival index scores with a progression in the GMFCS level.

The literature on the role of motor function on oral health of young adults with cerebral palsy is scant. One of the reasons for this is, perhaps, the fact that very often, a decreased motor function is accompanied by the use of a caregiver to provide oral health care [18]. It was for this reason that the sample in this study was restricted to individuals with GCMFS scores no greater than level III. Furthermore, this was also the reason for making sure that the participant being responsible for their own oral hygiene was an essential inclusion criterion.

There has been debate in the past over whether children and adolescents with cerebral palsy are effective rapporteurs of their quality of life $[17,18]$. There is ample evidence from medical literature to support that individuals with $\mathrm{CP}$ are effective rapporteurs of their own health-related quality of life $[3,4,22,26]$. There is also evidence to suggest that while qualitative data from individuals can be useful, in most cases, the use of validated questionnaires in individuals with $\mathrm{CP}$ has found to be a simple and effective measure of quality of life [3]. The decision to allow children to report their overall OHRQoL was also because the study was limited to children without intellectual disability who were attending regular schools.

The impact of cerebral palsy on the family is the one that has been extensively studied [7, 14, 28]. In keeping with previous research on OHRQOL in children and adolescents with $\mathrm{CP}$, the family impact scale was completed only by the parent $[14,18]$.

The results of the study need to be viewed considering certain limitations. The term cerebral palsy is an umbrella term used to classify different patterns of limited motor function. It was, therefore, necessary to restrict our study to only children with spastic cerebral palsy in keeping with the methodology used by similar studies assessing health-related quality of life. But, the limitation of this approach is that the findings may not be generalizable to all forms of CP. Further research with a larger sample is needed to confirm the generalize these findings to individuals with other forms of CP.

The study, however, has some important strength. The study reinforces the idea that function and activity participation are critical to the ability of young adults with cerebral palsy to lead independent and productive lives [3-5]. Clinically, the study also shows that young adults with $\mathrm{CP}$ 
are aware of their oral health challenges, and dentists in the $21^{\text {st }}$ century should be ready to meet these challenges.

\section{Conclusions}

With the limitations of the current study, we can conclude that motor function has a significant impact on both the oral health and the oral health-related quality of life of adolescents with spastic cerebral palsy. Further research on the topic is needed to prove the generalizability of these findings to all forms of cerebral palsy.

\section{Data Availability}

The data and additional materials are made available from the corresponding author upon request.

\section{Ethical Approval}

Ethical approval was obtained from the Institutional Review Board of the research center of the Riyadh Elm University (RC/IRB/2018/1054).

\section{Consent}

Consent to publish was obtained as part of the informed consent form from the parents of children in this study. Informed consent was obtained from the parents of the children, and assent was obtained from all participants before administration of the questionnaire or examination of the oral cavity.

\section{Conflicts of Interest}

The authors declare that they have no conflicts of interest.

\section{Authors' Contributions}

SCP conceptualized the study and carried out the data management and statistical data analysis. SFA, RNA, AAA, DNA, HFS, and NWA were responsible for data collection, patient follow-up, and patient management. All authors contributed equally to the preparation of this manuscript.

\section{Acknowledgments}

The authors are grateful to the research center at the Riyadh Elm University for logistical support and statistical consultation. The authors are also grateful to all staff and patients at the Prince Sultan Bin Abdulaziz Humanitarian City, without whose co-operation, this study would not have been possible.

\section{References}

[1] A. Levy-Zaks, Y. Pollak, and H. Ben-Pazi, "Cerebral palsy risk factors and their impact on psychopathology," Neurological Research, vol. 36, no. 1, pp. 92-94, 2014.

[2] A. Al-Asmari, K. Al Moutaery, F. Akhdar, and M. Al Jadid, "Cerebral palsy: incidence and clinical features in Saudi
Arabia," Disability and Rehabilitation, vol. 28, no. 22, pp. 1373-1377, 2006.

[3] T. Makris, D. Dorstyn, and A. Crettenden, "Quality of life in children and adolescents with cerebral palsy: a systematic review with meta-analysis," Disability and Rehabilitation, pp. 1-10, 2019.

[4] S. C. Wintels, D. W. Smits, F. Van Wesel, J. Verheijden, and M. Ketelaar, "How do adolescents with cerebral palsy participate? learning from their personal experiences," Health Expectations, vol. 21, no. 6, pp. 1024-1034, 2018.

[5] J. Jarl, A. Alriksson-Schmidt, and E. Rodby-Bousquet, "Health-related quality of life in adults with cerebral palsy living in Sweden and relation to demographic and disabilityspecific factors," Disability and Health Journal, vol. 12, no. 3, pp. 460-466, 2019.

[6] M. M. Eken, R. P. Lamberts, J. Du Toit, P. P. J. L. Verkoeijen, E. Kosel, and N. G. Langerak, "The level of accomplishment and satisfaction in activity and participation of adults with cerebral palsy and spastic diplegia," Journal of Orthopaedic Science, 2019.

[7] M. Burak and E. Kavlak, "Investigation of the relationship between quality of life, activity-participation and environmental factors in adolescents with cerebral palsy," NeuroRehabilitation, 2019.

[8] J. Goodwin, J. Lecouturier, A. Basu et al., "Standing frames for children with cerebral palsy: a mixed-methods feasibility study," Health Technology Assessment, vol. 22, no. 50, pp. 1-232, 2018.

[9] E. Roberts, M.-G. De La Peña, E. Ortibus, G. Molenaers, J. Deklerck, and H. Feys, "Functional outcomes in children and young people with dyskinetic cerebral palsy," Developmental Medicine \& Child Neurology, vol. 59, no. 6, pp. 634640, 2017.

[10] S. N. Drachev, T. Brenn, and T. A. Trovik, "Oral health-related quality of life in young adults: a survey of Russian undergraduate students," International Journal of Environmental Research and Public Health, vol. 15, no. 4, 2018.

[11] F. Lin, Y. Ye, S. Ye et al., "Effect of personality on oral healthrelated quality of life in undergraduates," The Angle Orthodontist, vol. 88, no. 2, pp. 215-220, 2018.

[12] A. H. Llanos, C. G. B. Silva, K. T. Ichimura et al., "Impact of aggressive periodontitis and chronic periodontitis on oral health-related quality of life," Brazilian Oral Research, vol. 32, Article ID e006, 2018.

[13] M. Schimmel, B. Leemann, P. Christou et al., "Oral healthrelated quality of life in hospitalised stroke patients," Gerodontology, vol. 28, no. 1, pp. 3-11, 2011.

[14] J. Abanto, T. S. Carvalho, M. Bonecker, A. O. Ortega, A. L. Ciamponi, and D. P. Raggio, "Parental reports of the oral health-related quality of life of children with cerebral palsy," BMC Oral Health, vol. 12, p. 15, 2012.

[15] J. Abanto, A. O. Ortega, D. P. Raggio, M. Bonecker, F. M. Mendes, and A. L. Ciamponi, "Impact of oral diseases and disorders on oral-health-related quality of life of children with cerebral palsy," Special Care in Dentistry, vol. 34, no. 2, p. 5663, 2014.

[16] K. Dalaie, M. Behnaz, Z. Khodabakhshi, and S. Hosseinpour, "Impact of malocclusion severity on oral health-related quality of life in an Iranian young adult population," European Journal of Dentistry, vol. 12, no. 1, pp. 129-135, 2018.

[17] E. Alvarenga, A. M. Silva, T. A. E. Da Silva, R. F. De Araujo, R. R. Prado Junior, and R. F. Mendes, "Oral health-related quality of life in caregivers of individuals with cerebral palsy: a 
case-control study," European Archives of Paediatric Dentistry, 2019.

[18] R. Akhter, N. M. M. Hassan, E. F. Martin et al., "Caries experience and oral health-related quality of life (OHRQoL) of children and adolescents with cerebral palsy in a low-resource setting," BMC Oral Health, vol. 19, no. 1, p. 15, 2019.

[19] E. A. El Ashiry, S. M. Alaki, and S. M. Nouri, "Oral health quality of life in children with cerebral palsy: parental perceptions," Journal of Clinical Pediatric Dentistry, vol. 40, no. 5, pp. 375-387, 2016.

[20] E. Longo, M. Badia, M. Begoña Orgaz, and M. Gómez-Vela, "Comparing parent and child reports of health-related quality of life and their relationship with leisure participation in children and adolescents with cerebral Palsy," Research in Developmental Disabilities, vol. 71, pp. 214-222, 2017.

[21] S. E. Reedman, R. N. Boyd, S. G. Trost, C. Elliott, and L. Sakzewski, "Efficacy of participation-focused therapy on performance of physical activity participation goals and habitual physical activity in children with cerebral palsy: a randomized controlled trial," Archives of Physical Medicine and Rehabilitation, vol. 100, no. 4, pp. 676-686, 2019.

[22] R. Power, M. Muhit, E Heanoy et al., "Health-related quality of life and mental health of adolescents with cerebral palsy in rural Bangladesh," PloS One, vol. 14, no. 6, Article ID e0217675, 2019.

[23] S. Q. Khan, N. B. Khan, and A. S. Arrejaie, "Dental caries. A meta analysis on a Saudi population," Saudi Medical Journal, vol. 34, no. 7, pp. 744-749, 2013.

[24] A. Wyne, A. Chohan, M. Al-Owaisi, and M. Al-Ahmari, "Oral hygiene and gingival health status of preschool children attending a University dental hospital," Pakistan Oral \& Dental Journal, vol. 23, no. 1, 2003.

[25] S. E. Reedman, R. N. Boyd, C. Elliott, and L. Sakzewski, "Participate CP: a protocol of a randomised waitlist controlled trial of a motivational and behaviour change therapy intervention to increase physical activity through meaningful participation in children with cerebral palsy," BMJ Open, vol. 7, no. 8, Article ID e015918, 2017.

[26] A. Walker, G. Colquitt, S. Elliott, M. Emter, and L. Li, "Using participatory action research to examine barriers and facilitators to physical activity among rural adolescents with cerebral palsy," Disability and Rehabilitation, pp. 1-12, 2019.

[27] J. Omura, M. Fuentes, and K. Bjornson, "Participation in daily life: influence on quality of life in ambulatory children with cerebral palsy," PMぬR, vol. 10, no. 11, pp. 1185-1191, 2018.

[28] N. S. Alves, V. P. Gavina, K. L. Cortellazzi, L. A. A. Antunes, F. M. Silveira, and A. V. Assaf, "Analysis of clinical, demographic, socioeconomic, and psychosocial determinants of quality of life of persons with intellectual disability: a crosssectional study," Special Care in Dentistry, vol. 36, no. 6, pp. 307-314, 2016. 